\title{
Cutaneous Nodules Arranged in a Sporotrichoid Distribution Over the Lower Extremity
}

\author{
Georgia Kyriakou, ${ }^{1}$ Eleftheria Vryzaki, ${ }^{1}$ Efthymia Gialeli, ${ }^{1}$ Sophia Georgiou ${ }^{1}$
}

1 Department of Dermatology, University General Hospital of Patras, Greece

Key words: keratoacanthoma, skin neoplasms, squamous cell carcinoma

Citation: Kyriakou G, Vryzaki E, Gialeli E, Georgiou S. Cutaneous nodules arranged in a sporotrichoid distribution over the lower extremity. Dermatol Pract Concept. 2020;10(1):e2020013. DOI: https://doi.org/10.5826/dpc.1001a13

Accepted: September 16, 2019; Published: December 31, 2019

Copyright: $\odot 2019$ Kyriakou et al. This is an open-access article distributed under the terms of the Creative Commons Attribution License, which permits unrestricted use, distribution, and reproduction in any medium, provided the original author and source are credited.

Funding: None.

Competing interests: The authors have no conflicts of interest to disclose.

Authorship: All authors have contributed significantly to this publication.

Corresponding author: Georgia Kyriakou, MD, MsC, cPhD, University General Hospital of Patras, Rion, Patras, P.O. 265 04, Greece. Email: geo_kyr@yahoo.gr

\section{Introduction}

Keratoacanthoma is a relatively common, low-grade cutaneous tumor that most commonly exhibits a self-limiting behavior. The lesions present as firm, sharply defined nodules with a central ulceration or prominent keratin plug. Pertinent physical findings are usually limited to the skin, with the majority of the lesions favoring the sun-exposed areas [1].

Histopathological characteristics highly resemble those of squamous cell carcinoma. Observation strategy is questionable and rarely practiced as a therapeutic means. Surgical excision of the entire lesion is a gold standard regimen $[1,2]$.

\section{Case Presentation}

A 65-year-old Caucasian man with an unremarkable medical history presented with a 1-month history of 2 painless nodules in a linear arrangement on his right leg (Figure 1, A and B). The patient reported that both lesions appeared 1 week after he sustained a penetrating plant thorn injury while fishing in fresh water. Prior to clinical admission he had received amoxicillin/clavulanic acid by mouth along with topical fusidic acid cream for 2 weeks without noticeable clinical improvement.
Cutaneous examination revealed 2 dome-shaped, well-demarcated, flesh-colored, nontender nodules with peripheral scaling and central crust, arranged in a sporotrichoid fashion over the pretibial area of the right leg. The proximal and the distal lesions were located inferior to the patient's knee and on the lower part of his right shin, respectively. Enlarged lymph nodes were palpated in the right inguinal area. He was afebrile and the overall physical examination was normal. Differential diagnosis included keratoacanthomas, cutaneous sporotrichosis, nocardiosis, Mycobacterium marinum infection, and Merkel cell carcinomas.

Routine hematological, biochemical, and immunological investigations were either negative or within normal range. Mantoux test was negative and chest $\mathrm{x}$-ray revealed no pathological findings.

Direct Gram stain, periodic acid-Schiff stain, ZiehlNeelsen and Giemsa stains as well as tissue cultures were found negative for mycobacteria, protozoa, fungi, and yeasts, including Nocardia brasiliensis, Sporothrix schenckii, Mycobacterium marinum, Actinomyces, and Leishmania, and positive for Serratia marcescens and Escherichia coli, which were considered bacterial contamination.

Two 6-mm punch biopsies were obtained for histopathological examination. Polymerase chain reaction for detection of 
mycobacteria was negative. Pending the biopsy results and given the clinical suspicion of lymphocutaneous sporotrichosis, the patient commenced a course of systemic antibiotics and antifungals.

Since the biopsy results were consistent only with pseudoepitheliomatous hyperplasia, and minimal improvement was noticed during the antibiotic and fungal treatment, we proceeded to complete surgical excision of both lesions. The biopsied tissue specimens were additionally stained with periodic acid-Schiff and Grocott-Gomori stains without identification of an infectious agent. Histopathology was consistent with keratoacanthomas in regression stage (Figures 2 and 3). There was no evidence of regional lymphadenopathy or relapse at the follow-up visit, conducted 6 months later.

\section{Conclusions}

Apart from infectious diseases, a number of neoplasms are uncommonly described to present in a sporotrichoid fashion. These include melanoma, squamous cell carcinoma, B-cell and T-cell lymphoma, Langerhans cell histiocytosis, peripheral nerve sheath tumor, and epithelioid sarcoma [2]. Nevertheless, in the majority of cases the tumors represented recurrent or metastatic disease rather than primary neoplasms. To our knowledge, a similar pattern of keratoacanthomas with each lesion being a primary tumor was first reported by Abudu and Cohen [2]. Our patient is the second case described, thus rendering it a necessity to take keratoacanthomas into consideration when encountering atypical cutaneous lesions arranged in a sporotrichoid pattern.

\section{References}

1. Kwiek B, Schwartz RA. Keratoacanthoma (KA): an update and review. J Am Acad Dermatol. 2016;74(6):1220-1233.

2. Abudu B, Cohen PR. Sporotrichoid keratoacanthomas: case report and review of neoplasms presenting in a sporotrichoid pattern. Cureus. 2018;10(8):e3196.
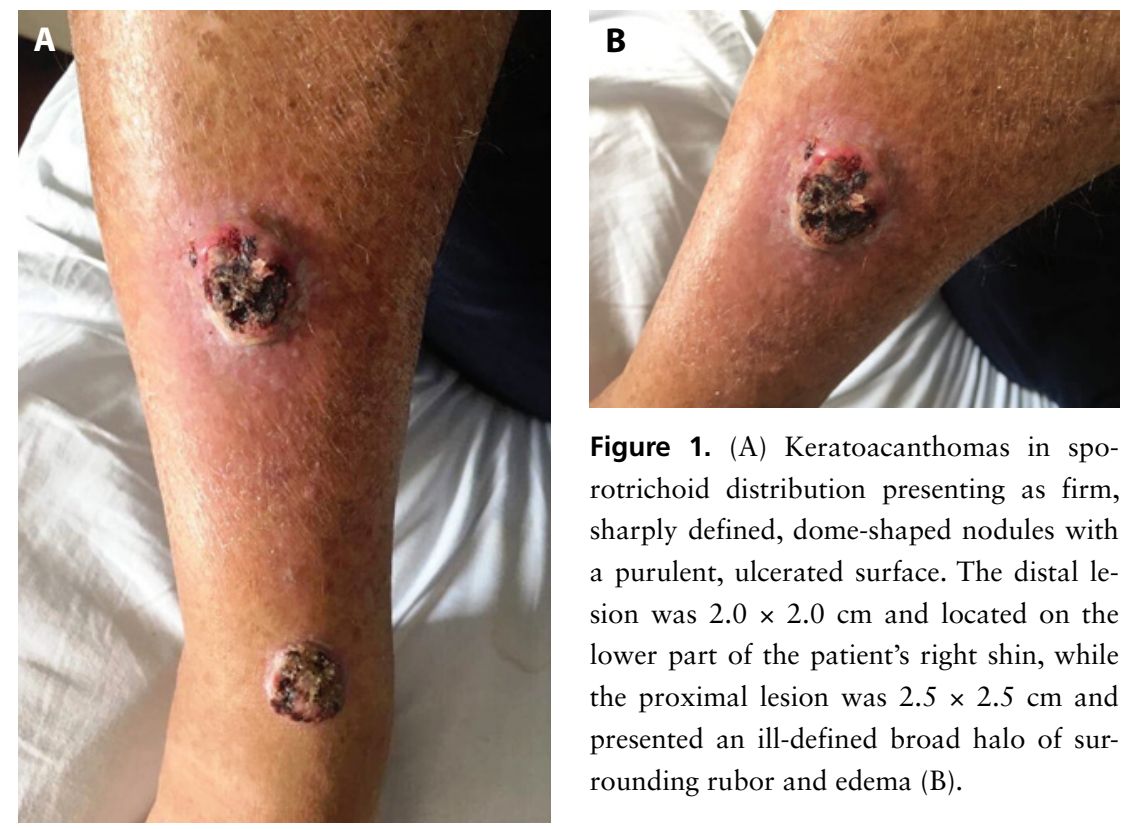

Figure 1. (A) Keratoacanthomas in sporotrichoid distribution presenting as firm, sharply defined, dome-shaped nodules with a purulent, ulcerated surface. The distal lesion was $2.0 \times 2.0 \mathrm{~cm}$ and located on the lower part of the patient's right shin, while the proximal lesion was $2.5 \times 2.5 \mathrm{~cm}$ and presented an ill-defined broad halo of surrounding rubor and edema (B).

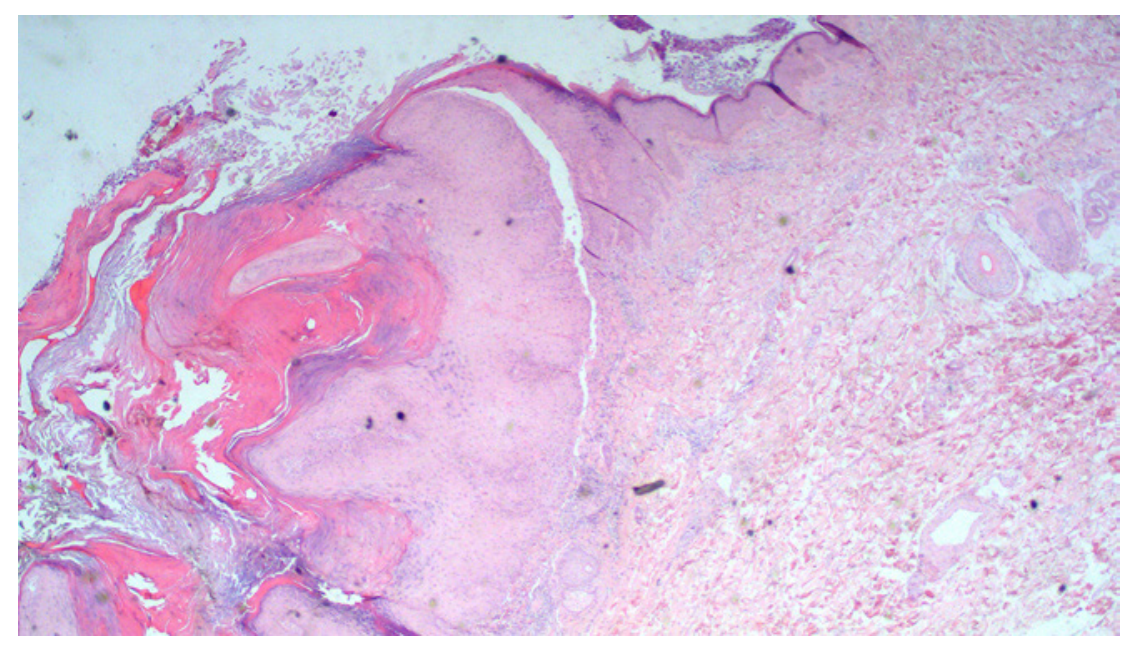

Figure 2. Histopathological examination of the biopsy specimen revealing a central crater filled with keratin (keratin plug), downward cupping of the epidermis, and hyperkeratosis with little or no parakeratosis $(\mathrm{H} \& \mathrm{E}, \times 40)$.

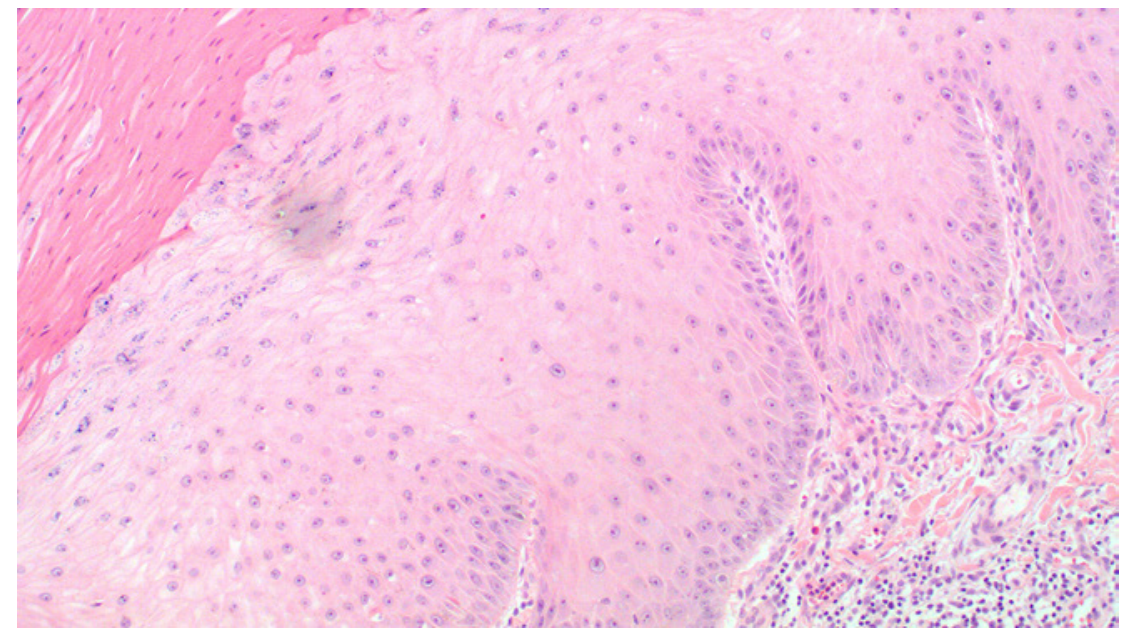

Figure 3. Keratin-producing squamous cells demonstrate minimal or absence of nuclear atypia. Marked inflammatory cell infiltrate is present around the lesion $(\mathrm{H} \& \mathrm{E}, \times 200)$. 